\title{
Investigation on matching characteristics of nuclear main pump guide vanes and annular casing
}

\author{
Xiaorui Cheng ${ }^{1,2} \odot \cdot$ Peng Wang ${ }^{1} \cdot$ Shuyan Zhang ${ }^{1}$
}

Received: 3 April 2019 / Accepted: 29 July 2019 / Published online: 5 August 2019

(c) The Author(s) 2019

\begin{abstract}
Based on the RNG $k-\varepsilon$ turbulence model, the multi-coordinate system is used to simulate the three-dimensional unsteady constant value of the nuclear main pump model under different axial and circumferential positions of the guide vane and the annular casing. By analyzing the external characteristics, pressure field and velocity field of different schemes and comparing the pressure pulsation at the impeller outlet, the flow of guide vane and the annular casing is studied through the axial and circumferential position changes in the vane and the annular casing. The results show that the axial distance between the center line of the guide vane and the center line of the outlet extension will increase the uneven distribution of pressure in the impeller outlet and the annular casing and the circumferential position of the guide vane will increase as well. When the center lines of the two are coincident, the pressure distribution in the impeller outlet and the casing is relatively uniform. The change in the axial and circumferential positions of the guide vane of the nuclear main pump has little effect on the vector distribution of the impeller outlet flow, and the influence of liquid flow in the interior of casing is large. The impeller outlet pressure pulsation increases as the axial distance between the guide vane outlet center line and the annular casing outlet center line increases. When the two center lines coincide, the impeller outlet pressure pulsation peak is the lowest.
\end{abstract}

Keywords Nuclear main pump $\cdot$ Impeller $\cdot$ Guide vane $\cdot$ Pressure pulsation

\section{Introduction}

The nuclear main pump is the only high-speed rotating equipment in the nuclear main pump circuit system. It has an irreplaceable role in the safe operation of nuclear power. Its stability and reliability will directly affect the normal operation of national defense and people's livelihood.

Technical Editor: Erick de Moraes Franklin, Ph.D.

Xiaorui Cheng

cxr168861@sina.com

Peng Wang

$1608744041 @$ qq.com

Shuyan Zhang

1804637589@qq.com

1 College of Energy and Power Engineering, Lanzhou University of Technology, No. 287 Langongping Road Qilihe District, Lanzhou City, Gansu Province, China

2 Key Laboratory of Fluid Machinery and Systems, Lanzhou University of Technology, No. 287 Langongping Road Qilihe District, Lanzhou City, Gansu Province, China
Bing et al. [1] compared the calculated values of the mixed flow pump under different turbulence models with the experimental values and learned that the corresponding turbulence model can accurately predict the external characteristics of the mixed flow pump under different flow conditions. Wang et al. [2] used the RNG $k-\varepsilon$ turbulence model and RANS equations in multiple reference frames to simulate the flow fields. Computational results reveal that the flow fields are unaxisymmetric in the impeller side chamber, and much more complex than that in an enclosed rotor-stator system. Cheng et al. [3] analyzed the dynamic and static head changes in the two flow lines in front of the blade suction in different cavitation conditions and found that the energy of fluid of nuclear main pump is provided by posterior segment of impeller, and the work capacity of blades decreases gradually from the shroud to the hub. Zhu et al., Xue et al. [4-6] optimized the blade and found that the axial and radial forces of the impeller are concentrated in the vibration of the low frequency region. Ni et al., Zhang et al. [7-9] discovered the dynamic and static interference is the main factor causing pressure pulsation in the nuclear main pump. The unsteady vortex flow in the pump will cause serious vibration of the 
pump. Su et al. [10] adopted the statistical method and spectral method to analyze the dimensionless pressure fluctuation intensity and radial force separately. The results show that the pressure fluctuation intensity of the guide vanes channel is stronger than that of the impeller channel and weaken as the temperature increases. Li et al. [11] found that the flow condition at the outlet is improved with a specific configuration of uneven guide blade, and the performance of multiple operating conditions is promoted. Effects of uneven guide blade on pressure fluctuation in different areas of model pump are of their own features. Li et al. [12] found that the different axial vibration amplitudes and the width of the flow channel have an effect on the axial dynamic characteristics through the study of the clearance of the nuclear main pump. Wang et al. [13] discussed the transient hydrodynamic characteristics of the nuclear main pump under a different volute eccentricity. Xu et al. [14] analyzed the pressure pulsation inside the nuclear main pump at different speeds and the uneven inflow under the corresponding speed and found that the low speed can reduce the vibration of the pump.

At present, there are many studies on the guide vanes of the nuclear main pump, but there are few studies on the matching characteristics of the guide vanes and the annular casing of the nuclear main pump. In this study, the five different axial positions of the guide vanes of the pumps are carried out, namely $x=1, x=0.5, x=0, x=-0.5, x=-1$. The full flow field simulation of the nuclear main pump was carried out based on the RNG $k-\varepsilon$ turbulence model in order to analyze the effect of the different axial placement positions of the guide vane.

\section{Geometry and numerical method}

\subsection{Model description and mesh generation}

Based on the AP1000 nuclear main pump, the nuclear main pump model with a scaling factor of 0.4 is selected as the

Table 1 Main structure parameters of nuclear main pump

\begin{tabular}{lll}
\hline Physical quantity & Parameter & Value \\
\hline Flow rate $\left(\mathrm{m}^{3} / \mathrm{h}\right)$ & $q_{\mathrm{v}}$ & 1145 \\
Head $(\mathrm{m})$ & $H$ & 17.8 \\
Rotational speed $(\mathrm{r} / \mathrm{min})$ & $N$ & 1780 \\
Impeller inlet diameter $(\mathrm{mm})$ & $D_{1}$ & 235 \\
Impeller outlet diameter $(\mathrm{mm})$ & $D_{2}$ & 340 \\
Impeller outlet width $(\mathrm{mm})$ & $b_{2}$ & 75 \\
Guide vane inlet diameter $(\mathrm{mm})$ & $D_{3}$ & 346 \\
Guide vane outlet diameter $(\mathrm{mm})$ & $D_{4}$ & 435 \\
Impeller blade number & $Z_{1}$ & 5 \\
Guide vane blade number & $Z_{2}$ & 18 \\
\hline
\end{tabular}

research object. The main design parameters of the model pump are shown in Table 1. The working medium is water. Figure 1 shows the structure of the nuclear main pump model. Figure 2 shows a cross-sectional view of the threedimensional fluid computing domain of the nuclear main pump model. The whole calculation fluid domain is divided into five parts, namely an impeller, a guide vane, an annular casing, and the inlet and outlet extension.

Due to the complexity of the flow inside the nuclear main pump and the complexity and large size of the structure, a non-structural tetrahedron mesh is adopted for the scaling of nuclear main pump model. Through the mesh-independent check and experimental verification, the total number of mesh is determined to be $6.25 \times 10^{6}$, and the number of mesh in each calculation domain is shown in Table 2. The computational domain mesh is shown in Fig. 3.

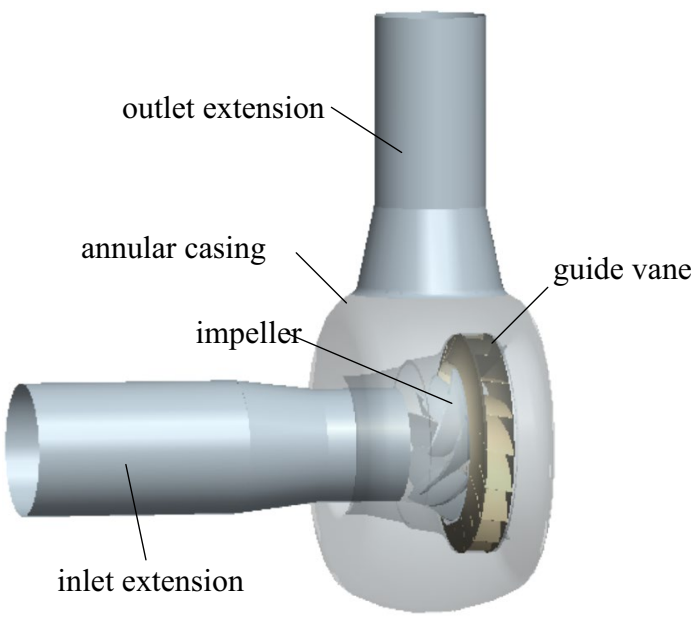

Fig. 1 Structure of the nuclear main pump model

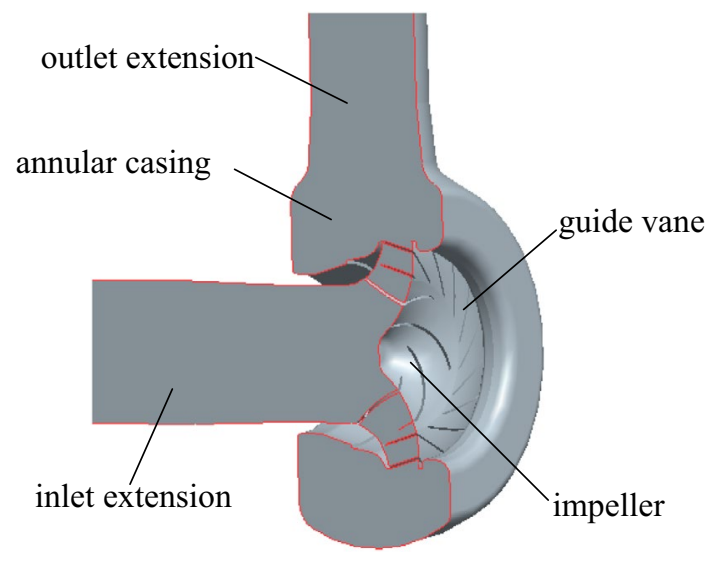

Fig. 2 A cross-sectional view of the three-dimensional fluid computing domain of the nuclear main pump model 
Table 2 Detailed mesh information

\begin{tabular}{|c|c|c|c|c|c|c|}
\hline Domain & Suction section & Impeller & Guide vane & Annular casing & Outlet section & Total \\
\hline Number of elements $\left(\times 10^{3}\right)$ & 810 & 1900 & 1510 & 1600 & 430 & 6250 \\
\hline
\end{tabular}

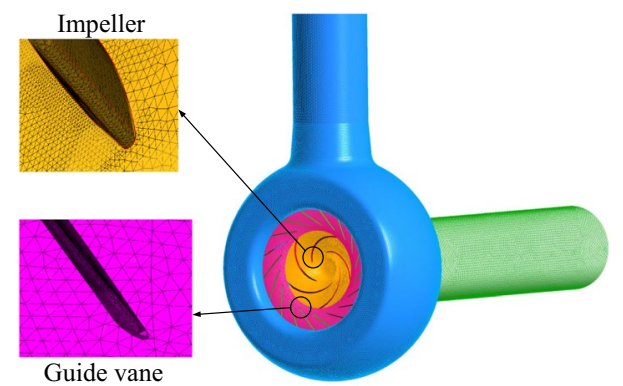

Fig. 3 Meshing of computational domain

\subsection{Numerical method}

Reynolds stress values were modeled using the RNG $k-\varepsilon$ turbulence model [15], which takes the flow separation and vortex flowing into account in the process of the pump flowing. At the same time, it can conduce to obtain high precision in its handling of the high strain rate and flow of the streamline. The pressure and momentum equations were coupled using the SIMPLEC algorithm with a high-resolution scheme. Usually, the numerical simulation of the nuclear main pump ignores the influence of the medium temperature change on the internal flow, so only the continuity equation and the momentum conservation equation are considered.

$\frac{\partial \rho}{\partial t}+\frac{\partial\left(\rho u_{i}\right)}{\partial x_{j}}=0$

$\frac{\partial\left(\rho u_{i}\right)}{\partial t}+\frac{\partial\left(\rho u_{j} u_{i}\right)}{\partial x_{j}}=\frac{\partial\left[\mu_{e}\left(\frac{\partial u_{i}}{\partial x_{j}}+\frac{\partial u_{j}}{\partial x_{i}}\right)\right]}{\partial x_{j}}-\frac{\partial p}{\partial x_{i}}+S_{i}$

where $\rho$ is the fluid density, and $x_{i}, x_{j}$ represent the respective coordinate components. $u_{i}$ and $u_{j}$ represent the average relative velocity components. $p$ is the pressure, and $S_{i}$ is the generalized source term.

In the RNG turbulence model, the transport equations for turbulent kinetic energy and dissipation rate are as follows:

$$
\begin{aligned}
& \frac{\partial(\rho k)}{\partial t}+\frac{\partial\left(\rho k u_{j}\right)}{\partial x_{j}}=\frac{\partial}{\partial x_{j}}\left[\alpha_{k} \mu_{\mathrm{eff}} \frac{\partial k}{\partial x_{j}}\right]+G_{\mathrm{k}}-\rho \varepsilon \\
& \frac{\partial(\rho \varepsilon)}{\partial t}+\frac{\partial\left(\rho \varepsilon u_{i}\right)}{\partial x_{i}}=\frac{\partial}{\partial x_{j}}\left[\alpha_{\varepsilon} \mu_{\mathrm{eff}} \frac{\partial \varepsilon}{\partial x_{j}}\right]+\frac{C_{1 \varepsilon} \varepsilon}{k} G_{\mathrm{k}}-C_{2 \varepsilon} \rho \frac{\varepsilon^{2}}{k}
\end{aligned}
$$

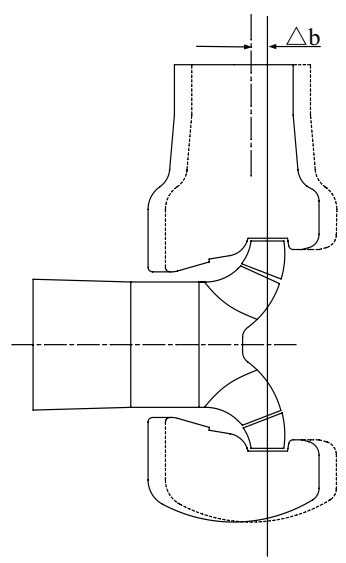

a axial position

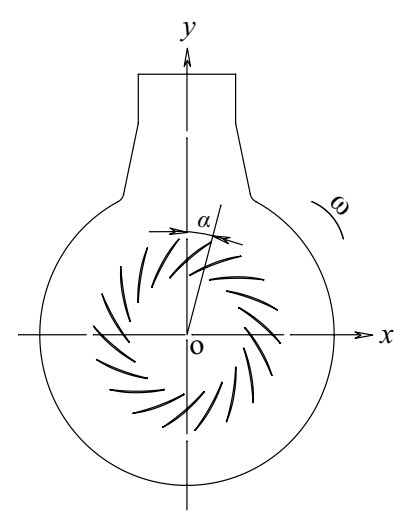

b circumferential position
Fig. 4 Matching scheme between guide vane and annular casing

The empirical constants in the equation are $C_{1 \varepsilon}=1.42$ and $C_{2 \varepsilon}=1.68, G_{\mathrm{k}}$ is the kinetic energy generation term, $\varepsilon$ is the turbulent dissipation rate, and $\mu_{\text {eff }}$ is the effective viscosity coefficient.

The calculation uses the three-dimensional steady NS equation and the RNG $k-\varepsilon$ turbulence model. The wall surface adopts the nonslip wall boundary condition. The standard wall function method is adopted in the area adjacent to the solid wall. The inlet boundary condition is designated as the velocity inlet, and the exit is the outflow. The convergence accuracy of the difference is $10^{-4}$. In order to study the pressure pulsation at the impeller outlet, the impeller is rotated through $3^{\circ}$ for a time step, and the time step is set to $\Delta t=0.00028568 \mathrm{~s}$. After 120 time steps, the impeller rotates for 1 week. In order to ensure the accuracy of the calculation results, the impeller is continuously rotated for six circles, and the pressure pulsation calculation result of the monitoring point $\mathrm{P}_{0}$ of the last cycle is selected for analysis [16].

\subsection{Scheme design}

As a prerequisite, to ensure the unchanged area of the annular casing and reasonable structure, five different guide vane axial placement positions are designed. The axial matching relationship is illustrated in Fig. 4a. This is the rule that when the outlet center line of the pump casing is on the left side of the guide vane, $\Delta b$ is ".+ " Otherwise, $\Delta b$ is " -" on the right side. Let $x=\Delta b / b$, where b is the width of the guide vane outlet, namely $x=1, x=0.5$, 
$x=0, x=-0.5, x=-1$, respectively. In order to study the influence of the change in the circumferential position of the guide vane on the pressure distribution of the annular casing, the circumferential angle of the guide vane $\alpha$ is defined as shown in Fig. 4b.

\subsection{Numerical method validations}

Figure 5 shows the nuclear main pump model experimental system, and Fig. 6 shows the comparison between the model pump head and efficiency experiment values and numerical calculation values. It can be seen from Fig. 6 that the numerical calculation head and efficiency values are higher than the experiment values under various working conditions. Under the design conditions, the head and efficiency values of the numerical simulation agree well with the experimental values. The head error does not exceed $2.7 \%$, and the efficiency error does not exceed $1.2 \%$. Under the non-design conditions, the calculated error of the head and efficiency is relatively large, but the head and efficiency values of the numerical calculation are consistent with the change trend of the experiment value. It shows that the model, mesh precision, and numerical method in the paper are suitable for this study.

\section{Results and discussion}

\subsection{Influence of axial position of guide vane on hydraulic performance of nuclear main pump}

Figure 7 shows the head and efficiency curves of the nuclear main pump in the five different axial positions of guide vanes. The relative flow is the ratio of the actual

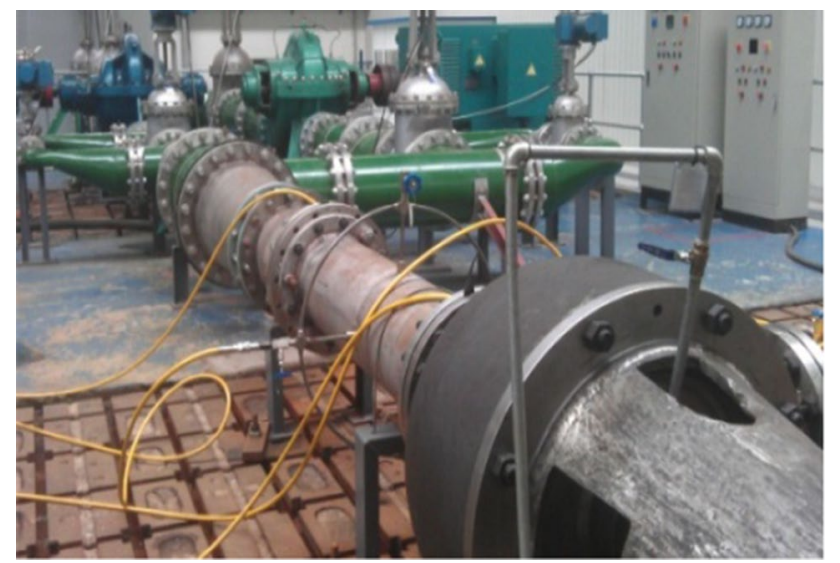

Fig. 5 Nuclear main pump model experiment system

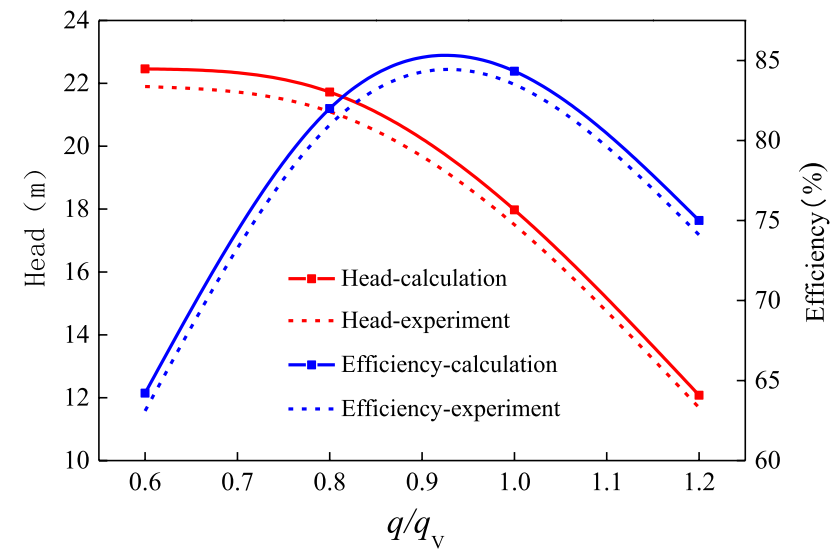

Fig. 6 Characteristic curves of model pump

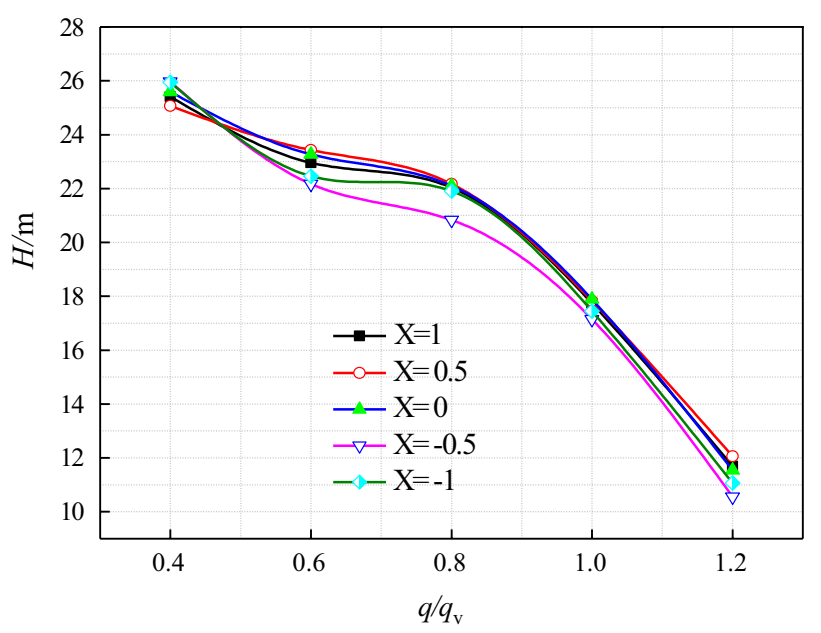

a head curves

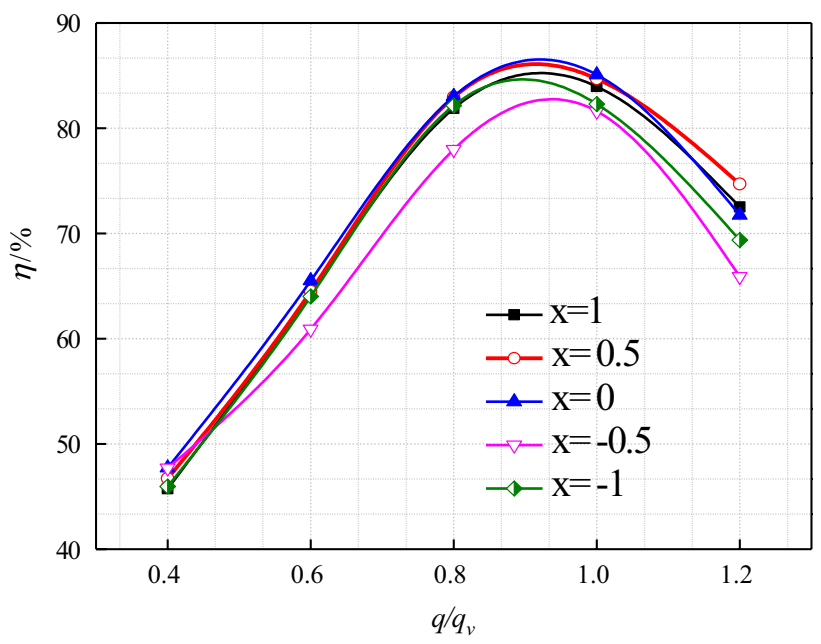

b efficiency curves

Fig. 7 Performance curves of pumps in different axial positions of guide vane 
flow $q$ to the design flow $q_{\mathrm{v}}$. It can be seen from the figure that the maximum change rate of the head is $4.1 \%$ and the maximum change rate of the efficiency is $4.0 \%$ owing to the change in the guide vane axial position. In the $x=0$ scheme, the head and efficiency of the nuclear main pump model reach the maximum at the same time, which indicates that the performance of the nuclear main pump is optimal when the center line of the annular casing outlet of the model pump coincides with the center line of the guide vane outlet. Under the conditions of $0.6 q_{\mathrm{v}}$ and $0.8 q_{\mathrm{v}}$, the head and efficiency trends are almost the same as the design conditions. That is to say, the head and efficiency increased initially, followed by a decrease, but then again increased as the center line of the annular casing outlet changes from the left to the right. When the center line of the outlet of the annular casing coincides with the center line of the outlet of the guide vane, the head and efficiency simultaneously reach the maximum value. When the flow is less than $0.4 q_{v}$, the change in the head and efficiency of the nuclear main pump model is small, namely the change in the axial position of the guide vane and the annular casing has little effect on the pump performance. Under the $1.2 q_{\mathrm{v}}$ condition, the head and the efficiency of the nuclear main pump model change greatly, so the change of the axial position of the guide vane has a great influence on the pump performance.

\subsection{Influence of axial position on internal pressure of nuclear main pump}

In view of the radial force of the impeller, the plane of the axis of the pump outlet extension is taken as the section A-A (as shown in Fig. 4a). The matching relationship between the axial direction and the circumferential position of the vane is investigated, and the internal pressure change in the main pump under steady pressure is analyzed. Figure 8 a shows the static pressure contours of section A-A at different axial positions of the guide vanes. It can be concluded from the figure that under the design condition, the pressure distribution of the impeller and the annular casing is significantly different with the change in the axial placement position of the guide vane. The static pressure value of the impeller from the inlet to the outlet is gradually increased and reaches the maximum at the outlet. Compared with the pressure contours of the impeller and the guide vane gap, it can be seen that as the axial distance $\Delta b$ between the center line of the guide vane outlet and the center line of the annular casing increases, the pressure distribution at the impeller outlet and the guide vane inlet is disordered. The pressure gradient changes significantly and consists in the high pressure zone. When the center line of the annular casing coincides with the center line of the guide vane outlet, the pressure at the impeller outlet and the guide vane inlet is relatively gentle and evenly distributed, which reduces the impact action of the fluid on the guide vane inlet. This is primarily because the geometry of the annular casing is unchanged, but the change in the axial position of the guide vane causes the flow direction and velocity of the impeller outlet to change greatly, which in turn affects the pressure distribution. According to the distribution of the pressure contours of the annular casing, as the axial distance $\Delta b$ increases, the distribution of the pressure disorder zone of the annular casing and the outlet extension changes significantly. When the center line of the annular casing coincides with the center line of the guide vane outlet, the kinetic energy of the liquid flowing out of the annular casing is greatly reduced and the flowing in the outlet extension is optimal. This is caused by the change in the axial position of the guide vane, which alters the velocity and direction of the flow at the outlet of the guide vane. Due to the change in the axial position of the guide vane, the flow that from the guide vane into the annular casing is not able to convert kinetic energy into pressure energy in time with the diffusion of the flow channel, and the larger liquid flow velocity aggravates the complex unsteady flow of the surrounding liquid, especially in the vicinity of the outlet of the guide vane.

It can be seen from Fig. $8 \mathrm{~b}$ that under the design condition, the pressure distribution of the impeller to the annular casing is completely different from the change in the circumferential position of the guide vane. But, the static pressure value of the impeller from the inlet to the outlet gradually increases and reaches the maximum at the impeller outlet. By comparing the pressure contours of the impeller and guide vane gap, it can be seen that with the change in the circumferential position of the guide vane, the pressure gradient at the impeller outlet and the guide vane inlet changes significantly, and both are in the high pressure zone. When the circumferential position of the guide vane is $\alpha=5^{\circ}$, the pressure on the inlet of the impeller and the guide vane changes gently, and the pressure distribution is relatively uniform, which can realize the free flow of the fluid, thereby reducing the impact loss of the fluid on the inlet of the guide vane. According to the pressure contours of the annular casing, as the circumferential position of the guide vane changes, the high pressure region of the annular casing changes greatly. The steady-state area of the pump outlet alters significantly because of the change in circumferential position of the guide vane. The change in the flow direction and velocity of the impeller outlet will inevitably affect the flowing state of the guide vane and the annular casing. Compared with the other guide vane circumferential position, when $\alpha=5^{\circ}$, the kinetic energy of the liquid flowing out of the annular casing is greatly reduced, and the liquid 


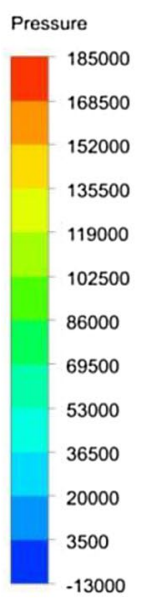

[Pa]

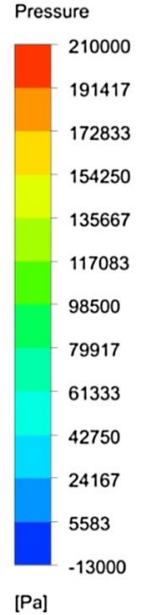

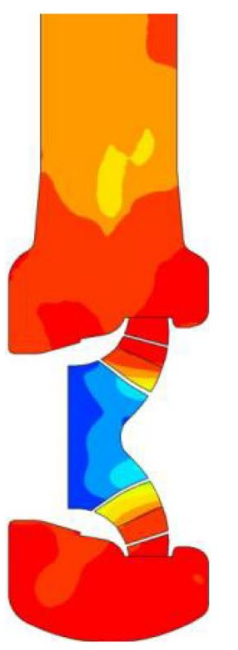

$x=1$

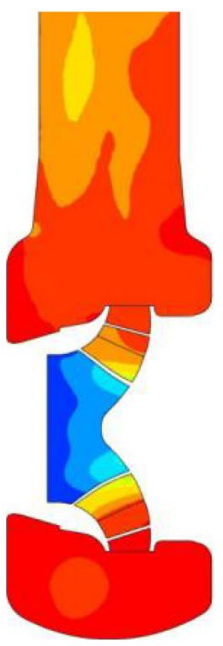

$x=0.5$

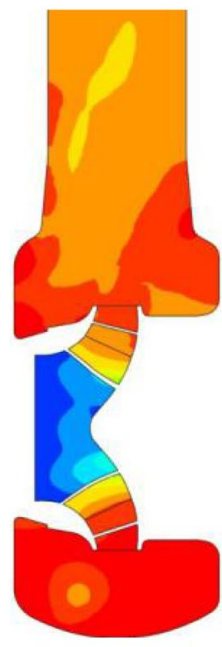

$x=0$

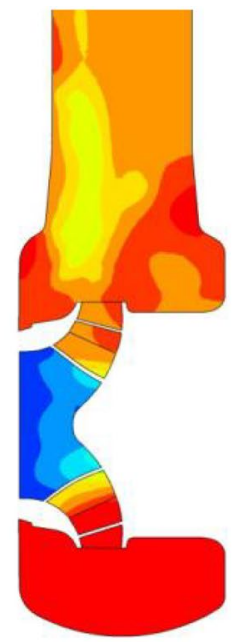

$x=-0.5$

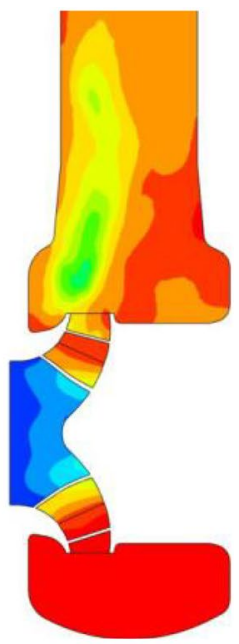

$x=-1$

a static pressure contours of A-A section under axial position
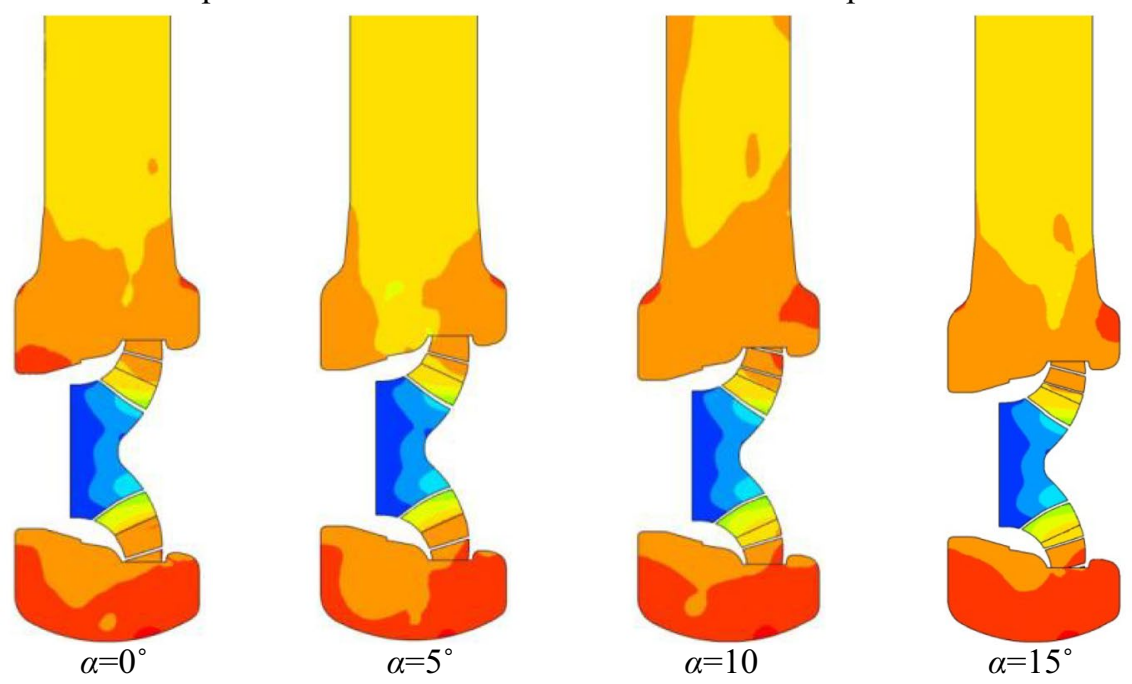

b static pressure contours of A-A section under different circumferential positions

Fig. 8 Static pressure contours of the guide vane and the volute

flow tends to be gentle. This indicates that the liquid flow at the impeller outlet can flow more uniformly into the annular casing along the guide vanes. It is beneficial for reducing the impact loss and unsteady turbulence in the process and uniformly converting the velocity of the liquid into pressure.

\subsection{Analysis of internal velocity field of nuclear main pump by matching guide vane with the annular casing}

Figure 9 shows the flow field of the A-A section in the axial and circumferential placement of different guide vanes. It can be observed in Fig. 9a, under the design condition, as the axial position of the vane changes, a pair of oppositely directed vortices exists in the annular casing on the far side of the pump outlet. The area also varies with the axial position of the guide vanes, and flow disturbances occur at the impeller outlet and the guide vane inlet. This is because the cross-sectional area of the annular casing on the far side from the outlet is small, and the flow at the outlet of the guide vane has a large kinetic energy. The narrow overflow area is not enough to smoothly convert the kinetic energy of the liquid flow into the pressure energy, causing the liquid flow to impinge on the wall surface of the casing. The change in the main flow direction of the liquid flow leads 

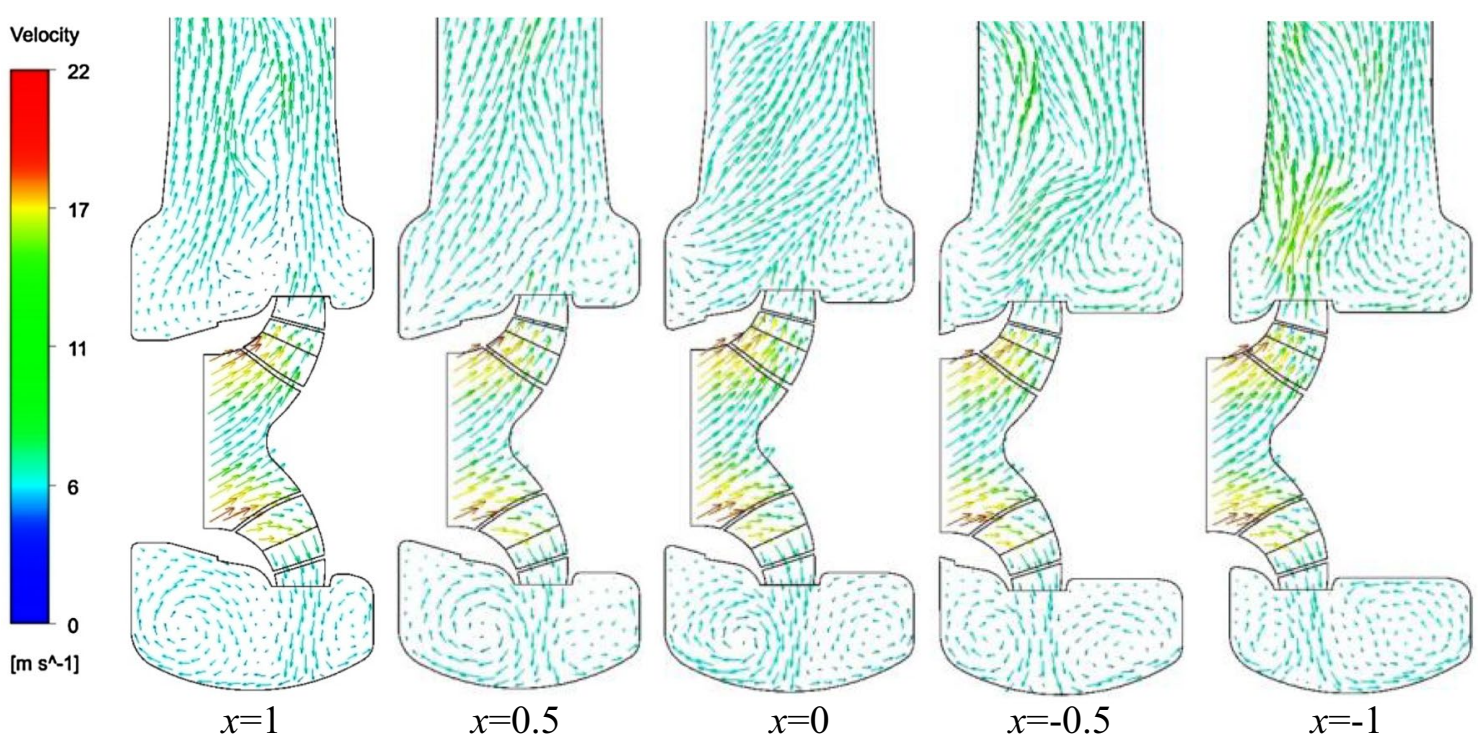

a velocity vector of A-A section under different guide vane axial position
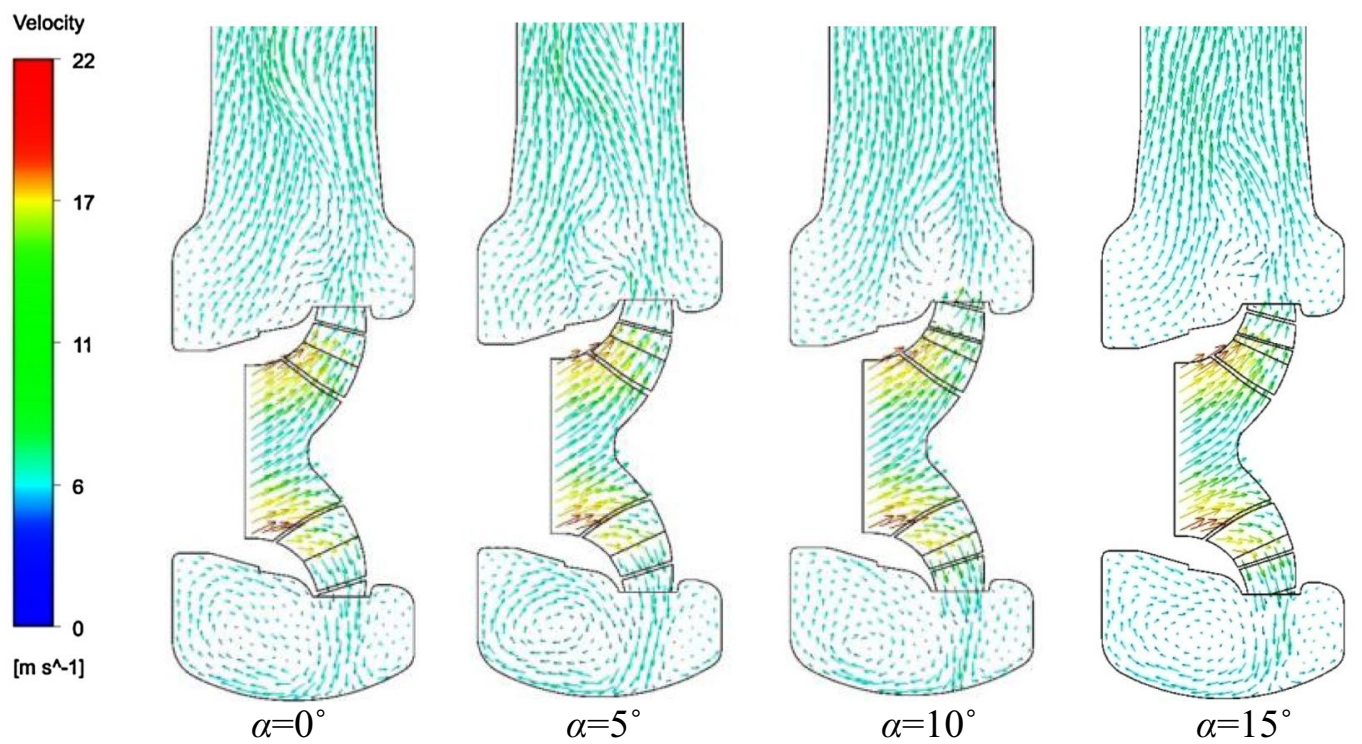

b velocity vector of A-A section under different guide vane circumferential position

Fig. 9 Guide vane and volute matching velocity vector

to the flowing near the wall surface of the annular casing falling off and forming a vortex opposite to the direction. The change in the axial position of the guide vane substantially changes the flow of the liquid into the main flow of the annular casing, which is the main cause of the change in the area of the vortex. In addition, it can be seen from the figure that the velocity vector distribution of the A-A section of the pump outlet is different. When the guide vane is located at the $x=0$ scheme, the flow of the annular casing is relatively uniform and stable, and there will be obvious disturbances and disordered movements in the annular casing when it is in other axial positions. This indicates that the change in the position of the guide vane has a great influence on the liquid flowing in the pump, which causes the flow of the liquid in the pump to be turbulent, and the turbulence in the annular casing affects the liquid flowing out of the impeller in turn.

It can be observed in Fig. 9b that under the design condition, as the circumferential position of the guide vane changes, a pair of oppositely directed vortices exists in the annular casing on the far side of the pump outlet. The area of the vortex has been changed but existed on the 


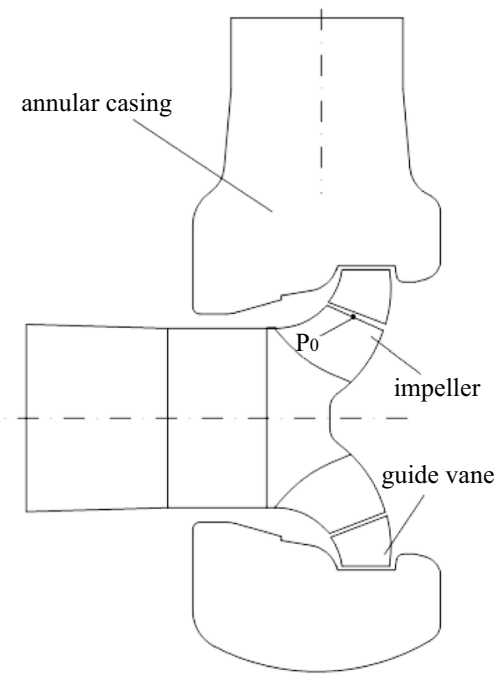

Fig. 10 Pressure fluctuation monitoring point layout

left. There is also a large velocity vector at the impeller outlet and the inlet of the guide vane. In addition, it can be seen from the figure that the fluid vector distribution on the A-A section of the pump outlet is significantly different. When the circumferential position of the guide vanes is $\alpha=10^{\circ}$, the internal flow of the annular casing is relatively uniform and stable, and when the guide vanes are in other circumferential positions, the flow in the annular casing will show obvious irregular movement. This is because the change in the circumferential position of the guide vane causes the flow direction of the impeller outlet to change. When $\alpha=10^{\circ}$, the flow of the impeller outlet can spread more naturally along the flow line of the guide vane, and the flow inside the annular casing is more uniform; other circumferential angles will have a greater impact on the flow at the impeller outlet, increasing the complexity of the flow field. By analyzing the influence of the position change in guide vane on the flow field, whether the guide vane is in the circumferential or axial position, the influence on the vector distribution of the fluid at the outlet of the impeller is small, but that in the annular casing is large. Therefore, the flowing in the annular casing is optimal when it reaches a suitable position.

\subsection{Influence of axial position of different guide vanes on pressure pulsation of impeller outlet}

In order to study the influence of different axial positions of the guide vanes on the impeller outlet pressure pulsation, a pressure monitoring point $\mathrm{P}_{0}$ is arranged at the center of the impeller outlet, as is shown in Fig. 10.
In order to intuitively reflect the pressure variation law in the nuclear main pump, the pressure coefficient is introduced and the pressure pulsation coefficient $C_{\mathrm{P}}$ is defined.

$C_{\mathrm{P}}=\frac{P-\bar{P}}{\frac{1}{2} \rho u_{2}^{2}}$

where $\mathrm{P}$ is the transient pressure of the monitoring point, $\bar{P}$ is the time-averaged pressure of one period of the impeller, $\rho$ is the density of the working medium, and $u_{2}$ is the circumferential speed of the intermediate flow line of the impeller outlet.

Figure 11 shows the pressure pulsation time domain of the impeller outlet $P_{0}$ at different axial placement positions. The abscissa is a period, and the ordinate is the pressure coefficient $C_{\mathrm{P}}$. It can be observed in the figure that the pressure distribution law at $P_{0}$ is basically the same with obvious periodicity. The pressure pulsation is mainly composed of main fluctuation and secondary fluctuation. The number of main fluctuation cycles is 5 , and the number of secondary fluctuation cycles is 1-2. This indicates that the number of principal fluctuation cycles is determined by the number of impeller blades, and the number of secondary fluctuations is determined by the number of impellers and guide vanes together. The $\mathrm{P}_{0}$ pressure pulsation coefficient is asymmetrically distributed, and the positive coefficient peak is smaller than the negative coefficient peak. The positive coefficient distribution range is larger than the negative coefficient. This is because the blade performs work on the fluid, and the pressure at the $\mathrm{P}_{0}$ is greater than $\bar{P}$ at different times, which is the main reason for the larger area of the positive coefficient range. When the blade passes the $\mathrm{P}_{0}$, the narrow flow area of the blade and the guide vane causes the jet phenomenon, bringing about a sudden drop in pressure and producing a negative coefficient. The constant interference of the blade and the guide vane is the root of the cyclical

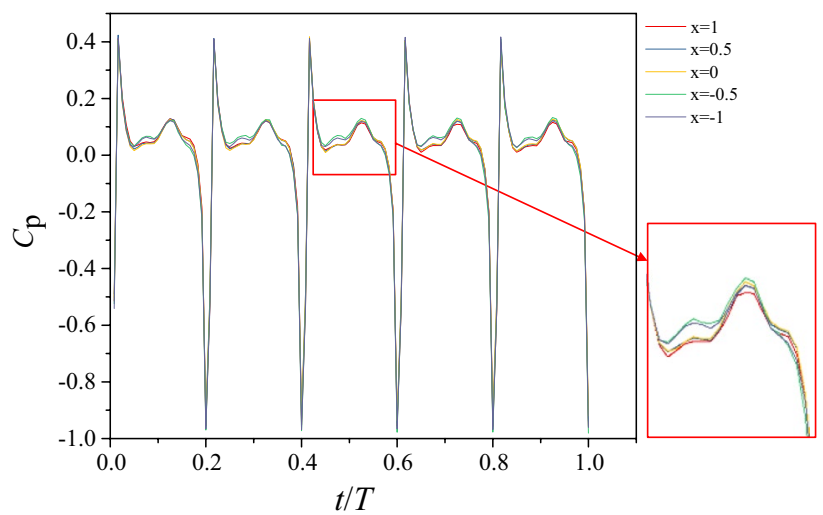

Fig. 11 Time-domain diagram of the pressure pulsation at the monitoring point $\mathrm{P} 0$ at the different axial positions 


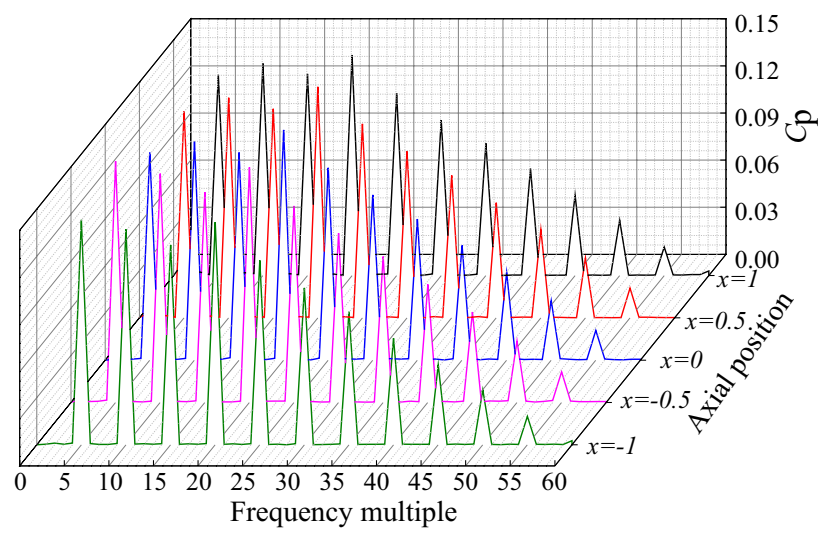

Fig. 12 Frequency-domain diagram of $\mathrm{P} 0$ at different axial positions

fluctuation of the pressure pulsation. As the model pump guide vane changes from the " $x=1$ " scheme to the " $x=-1$ " scheme along the axial position of the pump, the impeller outlet pressure pulsation peak changes, and the main peaks are $1.364,1.359,1.340,1.383$, and 1.382, respectively. It has the smallest peak value in the " $x=0$ " scheme. The distance between the center line of the annular casing outlet of the model pump and the center line of the guide vane outlet is appropriately decreased. The impeller pulsation peak value can be correspondingly reduced. It is helpful to improve the internal flow of the main pump and reduce the vibration inside the pump and prolong the service life of the pump shaft.

Figure 12 shows a pressure pulsation frequency-domain picture of the $\mathrm{P}_{0}$ of the guide vane at distinct axial positions. The time-domain picture of five different axial placement positions is obtained by fast Fourier transform (FFT) to obtain a pressure pulsation frequency-domain picture. The ordinate is the pressure pulsation peak corresponding to each frequency domain. It can be seen from the figure that the main frequency of the $\mathrm{P}_{0}$ pressure pulsation is $145.83 \mathrm{~Hz}$ at different axial positions, which is 5 times of the frequency conversion $f_{\mathrm{n}}=29.17 \mathrm{~Hz}\left(f_{\mathrm{n}}=n / 60, n\right.$ represents the rotation speed), just coincident with the blade frequency $145.83 \mathrm{~Hz}$ is consistent $(f=n Z / 60$, where $n$ is the speed, and $Z$ is the number of blades), and other pulsating peaks appear at integer multiples of the blade frequency, showing a periodic decrease. It is indicated that the axial position of the guide vane changes, and the pressure pulsation at the impeller outlet is mainly determined by the blade frequency; at the same time, the change in the axial position of the guide vane only affects the peak value of the pressure pulsation. It can also be concluded that the blade frequency is the main excitation frequency of the pressure fluctuation of the nuclear main pump model. As is seen from the figure, the pressure pulsation of the guide vanes at the position of $x=0$ is lower than other positions under the same frequency multiple, which demonstrates that the guide vanes can reduce the dynamic and static interference between the impeller and the guide vanes at the position of $x=0$. It provides a valuable reference for reducing fluid vibration and hydrodynamic noise in the pump.

\section{Conclusions}

1. As the axial distance $\Delta b$ increases and the circumferential position changes, the unevenness of pressure distribution in the impeller outlet and the annular casing will increase. When the center line of the annular casing outlet coincides with the guide vane outlet center line, the pressure distribution at the impeller outlet and the guide vane inlet is relatively uniform. The flowing in the annular casing is relatively stable, thereby reducing the impact loss of the fluid on the guide vane inlet.

2. The axial and circumferential positions of the guide vane of the nuclear main pump have little influence on the vector distribution of the fluid at the impeller outlet, and they have a great influence on the fluid flow inside the annular casing. When the guide vane is in the proper position, the flowing in the annular casing is optimal.

3. The impeller outlet pressure pulsation increases as the axial distance between the guide vane and the annular casing outlet center line increases. When the annular casing outlet center line coincides with the guide vane outlet center line, the impeller outlet peak value of the pressure pulsation is at a minimum. Appropriately changing the position of the guide vane can reduce the peak pressure pulsation of the impeller outlet, improve the internal flow of the nuclear main pump, and reduce the vibration of the pump, which is beneficial to the safe and reliable operation of the nuclear main pump.

Acknowledgements This work was supported by the National Natural Science Foundation of China (No. 51469013).

Open Access This article is distributed under the terms of the Creative Commons Attribution 4.0 International License (http://creativeco mmons.org/licenses/by/4.0/), which permits unrestricted use, distribution, and reproduction in any medium, provided you give appropriate credit to the original author(s) and the source, provide a link to the Creative Commons license, and indicate if changes were made.

\section{References}

1. Bing H, Cao S, Wang Y (2013) Influence of turbulence model on performance prediction of Mixed-flow Pump. Trans Chin Soc Agric Mach 44(11):42-47 
2. Wang X, Wang C, Li Y (2009) Numerical study of flow characteristics in the impeller side chamber of centrifugal pump. Trans Chin Soc Agric Mach 40(4):86-90

3. Cheng X, Fu L, Bao W (2018) Effect of cavitation flow on energy transfer in nuclear main pump. J Drain Irrig Mach Eng 36(5):369-376

4. Zhu R, Cai Z, Wang X (2018) Cavitation characteristics and dynamic characteristics of a nuclear main pump based on the layer constraint of blades. J Vib Shock 37(10):35-42+79

5. Zhu R, Li X, Yuan S (2012) Effect of annular casing outlet contraction angle on hydraulic performance of main nuclear reactor pump. Nucl Power Eng 33(2):97-103

6. Xue M, Piao Y (2012) Numerical simulation on separation control by filleting casing tongue of after-burning fuel pump. J Aerosp Power 27(12):2799-2804

7. Ni D, Yang M-G, Gao B (2017) The internal correlations between unsteady flow and pressure pulsations in a nuclear reactor coolant pump. J Eng Thermophys 38(8):1676-1682

8. Ni D, Yang M, Gao B (2017) Numerical study on the effect of the diffuser blade trailing edge profile on flow instability in a nuclear reactor coolant pump. Nucl Eng Des 322:92-103

9. Zhang N, Yang MG, Gao B, Li Z, Ni D (2015) Experimental investigation on unsteady pressure pulsation in a centrifugal pump with special slope volute. J Fluids Eng 29(10):4231-4238

10. Su S, Wang P, Xu Z (2017) Study on pressure fluctuation and radial force during startup of reactor coolant pump. Nucl Power Eng 38(3):110-114
11. Li J, Wang X, Zhou F (2014) Influence of uneven guide blade on performance and pressure fluctuation of nuclear model pump. Fluid Mach 42(9):19-24

12. Li Z-1, Xu S-1, Guan Z-q (2018) Dynamic analysis of the axial vibration of the impeller in a reactor coolant pump induced by the radial leakage flow. Chin J Comput Mech 35(4):458-465

13. Wang P, Yuan S-q, Wang X-1 (2015) Volute eccentricity effect on transient dynamic characteristics of nuclear main pump. J Vib Shock 34(22):13-18

14. Xu R, Long Y, Wang D (2018) Effects of rotating speed on the unsteady pressure pulsation of reactor coolant pumps with steamgenerator simulator. Nucl Eng Des 333:25-44

15. Kurokawa FA, Correa L, Queiroz RABD (2018) Numerical simulation of 3D unsteady turbulent free surface flows using \$\$Ikappa - IIvarepsilon\$\$ $\$$ - $\varepsilon$ model and ADBQUICKEST scheme. J Braz Soc Mech Sci Eng 40(4):202

16. Lu Z, Wang C, Qiu N (2018) Experimental study on the unsteady performance of the multistage centrifugal pump. J Braz Soc Mech Sci Eng 40(5):264

Publisher's Note Springer Nature remains neutral with regard to jurisdictional claims in published maps and institutional affiliations. 